\title{
La interconsulta en salud mental infantil de niños recién nacidos con alto riesgo
}

RESUMEN: El avance técnico y de conocimientos en el campo de la Neonatología ha logrado que muchos recién nacidos (R.N.) sobrevivan a enfermedades que antes eran impensables de superar.

Este progreso ha supuesto a su vez, que se den procesos y problemáticas clínicas, que requieren nuevas formas de intervención, y de modelos interdisciplinares de actuación, sobre las distintas partes afectadas (bebés, padres, personal, etc).

Derivado de todo ello, las actuaciones en Salud Mental Infantil, van a jugar un papel muy importante sobre los procesos e intercambios afectivos primarios y los efectos que sobre las familias supone la presencia de su bebe gravemente enfermo, sus vivencias, sus ansiedades, reacciones, etc que requerirán una elaboración y apoyo terapéutico desde el campo de la salud mental.

Recogemos en este trabajo nuestra experiencia en la interconsulta con el Servicio de Neonatología desde nuestra Unidad de Salud Mental Infantil actuando tanto sobre el R.N. como sobre los padres y el personal.

PALABRAS CLAVE: Interconsulta de S.M.I. en Neonatología /R.N. de alto riesgo/ Programa padres-niño-personal./ Intervenciones psicoterapeúticas.
SUMMARY: The technical and theoretic in the field of Neonatology has caused that many newborn children survive to deseases that were imposible to overcome before now.

This progress has meant at the same time that clinical processes and problems emerge requiring new forms of management and new interdisciplinary models of approach to the different parts concerned (babies, parents, staff, and so on).

The outcome is that interventions in Child Mental Health are prone to play a very important role in primary afects and interchange processes, and in the effects derived from the presence of the seriously ill babies upon the families, as well as their experiences, their anxieties, their reactions, etc., that will need a working out and a therapeutic support from the mental health area.

In this paper we point out our experience in consultation-liaison with the Neonatology Service from our Child Mental Health unit, operating on the newborn as much as on the parents and the team members.

KEY WORDS: Consultation-liaison of Child mental Health in Neonatology / High risk newborn / Parents-childrenteam programme / Psichoterapeutic interventions. 
INFORMES

\section{Introducción.}

Recogemos la experiencia desarrollada en nuestra Unidad de salud Mental Infantil (U.S.M.I.) del Hospital Materno Infantil Juan Canalejo de A Coruña en la interconsulta con el servicio de neonatología.

Esta actividad se realiza junto con todos aquellos otros servicios que se ocupan de los niños recién nacidos (R.N.) que padecen graves enfermedades o cuadros discapacitantes.

Nuestra U.S.M.I., está ubicada en dicho hospital dependiendo del Servicio de Salud Mental. Tiene como funciones las propias de la atención a niños de 0 a 16 años, tanto ambulatoriamente con la población que nos corresponde del área sanitaria, como de la interconsulta e ingresos psiquiátricos del hospital materno infantil.

\section{Material y Métodos.}

Tomamos como muestra la casuística recogida durante el año 1996. Exponemos el $\mathrm{n}^{\mathrm{o}}$ de casos atendidos, la enfermedad que padecen, la expresión sintomática psíquica de los padres, las intervenciones realizadas dentro del programa padres-niño de apoyo psicoterapéutico y otras intervenciones de nuestro Servicio (ver tablas de 1 a 4). Nuestra actuación se enmarca dentro de una concepción de trabajo interdisciplinar (neonatología, rehabilitación infantil, servicios sociales, etc.) .El trabajo se realiza bien sea directamente sobre el caso o bien orientando o supervisando al profesional que lleva al bebé.

Se intenta evitar las peticiones de interconsulta en forma de "trabajo a la cadena" o sin intercambios directos y continuados, para poder realizar un trabajo basado en la idea de equipo e interdisciplinar, en el que los conocimientos de los diferentes campos confluyan, interactúen y se articulen para dar una respuesta al problema concreto y a la realidad especifica del caso por caso, abarcando e incluyendo tanto al niño como a la familia y al personal institucional que se ocupa del R.N.

Nuestra observación se hizo partiendo de una visión en la que los presupuestos teóricos que sostienen nuestra práctica clínica plantean la cuestión de la realidad psíquica, no reducida a formas mecanicistas, sino que introducen la dimensión del sujeto y de lo simbólico como fundamental para la comprensión de la conducta y de los fenómenos de salud mental.

No creemos que haya una observación sin preconcepciones conceptuales, que son las que van a marcar la propia observación y lo observado. Cierta falsa objetividad lleva a confundir lo aparente con lo real, las causas con los efectos, el acto con el pensamiento, etc, etc, y a que en último término se borre al propio sujeto como tal entidad de observación y de existencia.

Planteamientos globales.

Ante todo R.N. de alto riesgo, nos vamos a encontrar una problemática que, aunque particular de cada caso, podemos sintetizarla en los siguientes puntos:

-Situación de crisis familiar ante el nacimiento del R.N. con graves trastornos que ponen en juego su vida y su futuro desarrollo. 
INFORMES

-Momento de desorganización e incertidumbre ante la realidad traumática que está presente.

-Ruptura de los investimentos e intercambios afectivos primarios.

-Dificultad para los padres de efectuar de manera natural el proceso de creación y representación del bebé imaginario.

-Dificultad para desarrollar las funciones parentales y las vivencias e intercambios naturales, mediatizados por el riesgo de muerte, la incertidumbre, los tratamientos y la hospitalización a que está sometido el bebé.

-Trastornos en el desarrollo psicofísico del R.N. consecuencia de su enfermedad.

\section{Objetivos.}

A un nivel general, los principios que marcan nuestras intervenciones los podemos resumir en:

- Estimular y activar el desarrollo bio-psico-social armónico del niño.

- Elaboración y apoyo de la crisis parental.

- Recuperación y elaboración de las funciones parentales y de los intercambios afectivos primarios.

- Actuación interdisciplinar.

\section{Expresión clínica:}

La expresión clínica y las reacciones que podemos observar en los padres y en el R.N. de alto riesgo, no se pueden reducir a fórmulas rígidas, homogéneas y estandarizadas, porque tanto en el niño como en los padres y a nivel institucional, esta expresión clínica dependerá de múltiples factores que van desde la propia estructura psíquica, hasta los diversos cuadros clínicos que se presentan. Sin embargo podemos describir algunos rasgos comunes con los que nos encontramos mas frecuentemente:

- En los padres:

Ante su bebé gravemente enfermo algunos padres pueden presentar sentimientos de angustia e incertidumbre, fracaso y frustración, sentimiento de culpa, impotencia e incapacidad. Puede llevarlos asimismo al rechazo y negación de la aceptación del R.N. (no verlo, no tocarlo, ambivalencia...)

La expectativa puesta en su bebé y las fantasías abiertas en el encuentro esperado quedan frustradas y postergadas ante la incertidumbre y evolución de la enfermedad. El R.N. es sentido y vivido "como del hospital" (estado de dependencia, cuidados, tratamientos, etc). El niño "es" para los padres un diagnostico. Su cuerpo tiende a ser, no un lugar de satisfacción, sino un lugar de enfermedad, dolor y anomalía. No es un niño sentido e investido como gozoso, sino medicalizado, enfermo, cuerpo dañado o mortífero. El bebé no existe para los padres como un nombre propio que lo inscribe en la historia familiar. La atribución de un diagnóstico y la enfermedad borra su esencialidad como sujeto.

En algunos casos aparecen sentimientos ambivalentes de vida y muerte, inaceptables y culposos, versus mecanismos de reparación como superprotección compensadores. 
INFORMES

Puede darse la Maternidad blanca, como mecanismo inconsciente de defensa, quedando en una especie de anestesia emocional, en tanto falsa adaptación y aparente aceptación de los hechos, sintiéndose insensible, apática o indiferente.

Hay una ruptura de la continuidad relacional madre-niño con lo que esto conlleva de ruptura de los lazos e intercambios afectivos primarios, alterados y condicionados por la hospitalización y los tratamientos (aislamiento, catéteres, sondas, aparatos, etc), lo cual conlleva que no pueda recrearse el bebé imaginario, ilusionado y fantaseado..., ocupando este lugar, los temores, ansiedades, incertidumbres, vivencias de enfermedad y sufrimiento del bebé.

El alumbramiento en tanto fenómeno simbólico y cultural de los ritos e intercambios sociales y de la historia familiar queda suspendido en el tiempo, relegado y dilatado hasta su curación y alta, con lo que todos aquellos acontecimientos que forman parte de los rituales culturales y sociales propias del nacimiento van a quedar pendientes y postergados.

- En el niño:

Los trastornos derivados de su grave enfermedad, llevan consigo la posible falla de los mecanismos de intercambio, estimulación e interactivos madre-niño y lo que de ello puede derivarse: la deprivación afectiva y ruptura del vínculo primario.

Existen igualmente fenómenos derivados de la hospitalización y de los tratamientos que está recibiendo (dolor, hipersensibilidad...). Hemos de tener en cuenta que el R.N. percibe los estímulos nociceptivos mas intensamente que el adulto y sus mecanismos de defensa por contra, están menos desarrollados.

Asimismo nos vamos a encontrar con la fractura de la homeostasis y del flujo de los intercambios madre-niño habituales (sueño-vigilia, hambre-saciedad, llamadarespuesta, caricias, etc), es decir, todos aquellos intercambios propios de las relaciones primarias y de la inscripción del bebé en el deseo del adulto.

El niño por su grave enfermedad va a quedar sometido a agresiones tanto internas como externas ... "su cuerpo en vez de ser un lugar de goce en donde la autonomia vendrá a inscribirse, se convierte en lugar de dolor.... El conjunto de modificaciones intra y extra personales motivadas por la enfermedad modifica fundamentalmente la estructuración del sujeto ...." (V. Mira).

Dada la situación en que se encuentra el niño hay una ruptura del investimento de las funciones como "cuerpo hablado" (palabras, contactos, caricias, aseo, etc) dificultado y mediatizado por el tratamiento, que hace prioritario y puede reducirlo a las necesidades y cuidados fisicos en el plano de la mera necesidad biológica, desinsertada de un orden simbólico y afectivo.

Como ya antes expusimos al referirnos a los padres, el niño queda suspendido en el tiempo de su historia familiar y social, en el orden de los rituales y formas culturales del nacimiento, quedando relegado, en la espera de una resolución de su enfermedad, a ser todavía alguien determinado por la incertidumbre de ser y la posibilidad de existir. 
INFORMES

\section{Criterios de actuaciones en S.M.I.}

El eje articulador de toda intervención debe basarse en el trabajo interdisciplinar, en el sentido ya referido en la introducción, no como suma de actuaciones a la cadena, sino como intersección de conocimientos e intervenciones que confluyan en la mejor calidad de atención en lo particular de cada caso.

La derivación a Salud Mental Infantil debe ser una medida mas, dentro de un conjunto de necesidades y actuaciones, que no esté desgajada, ni aislada y que abarque tanto al niño como a los padres y al personal asistencial.

\section{- Con los padres:}

Como primer paso, toda información que se les de acerca de su hijo debe ser esclarecedora, sencilla, sin dramatizaciones, ni formulaciones de criterios desvalorizativos, o prejuicios premonitorios, negativos o vejatorios y culpabilizadores o moralizantes. Se deberá evitar igualmente las formas paternalistas y superprotectoras, o por el contrario ofensivas y displicentes.

Se favorecerá la presencia e intercambios de los padres con el bebé, ayudando a elaborar las fantasías y vivencias puestas en el R.N. (elaboración de pérdida, duelo, ansiedades, culpabilidad, etc).

Según sea la problemática presentada por los padres y las necesidades que precisen, se realizará un trabajo psicoterapéutico específico (grupos parque, psicoterapia de grupo, individual, etc)

\section{- Con el niño:}

Debemos favorecer las relaciones y vínculos padres-niño que permitan los intercambios y estímulos que son habituales en las relaciones primarias (contactos físicos, olores, caricias, palabras, etc) estos intercambios deben personalizarse en cada R.N. en base a las costumbres y realidad social, cultural, etc de cada caso.

En el tratamiento hospitalario deben reducirse en lo posible los estímulos unimodales mecánicos y desapacibles (pitidos, sonidos intensos, alarmas, aislamiento ...) Uno de los efectos que debemos prevenir son las secuelas y trastornos de la deprivación afectiva; síndrome de hospitalismo; etc que puedan presentarse ante la separación prolongada y el estado en que se encuentra el niño.

Es importante aplicar técnicas de observación de bebés (Brazelton; psicodinámicas....) que permitan conocer y valorar el nivel madurativo y los trastornos en el desarrollo psicomotor y relacional.

En aquellos casos que la presencia de los padres deba restringirse por razones terapéuticas, o bien haya problemática socio-familiar, debe realizarse el maternaje terapéutico con el bebé, a través del personal de enfermería y auxiliar como padres sustitutivos, que atenúen la fractura en los intercambios afectivos y proporcionen los 
vínculos, relaciones y estímulos básicos indispensables para un armónico desarrollo psico-orgánico, no reduciendo al R.N. a meros cuidados físicos.

- Interactuaciones padre-bebé:

Uno de los elementos clave en la problemática de estos casos es la ruptura de los intercambios padres-bebé y las consecuencias que de ello se deriva. De aquí la importancia de conocer y actuar sobre estos elementos interactuantes.

Ya hemos señalado la necesidad de asegurar los investimentos y vínculos afectivos que en estos casos están alterados y condicionados por la enfermedad, las secuelas y el estado en que se encuentra el bebé. En este sentido debemos estar atentos y escuchar las vivencias de los padres, sus sentimientos, ansiedades, fantasías, preocupaciones, opiniones, etc, etc con el fin de elaborar estas, e impedir sus repercusiones negativas.

Asimismo hemos de favorecer la reconstrucción de la historia y el deseo prefigurado que en condiciones normales se hace de forma natural y espontánea (nombre, ilusiones, aspiraciones, fantasías puestas en el bebé ....). Todo esto es lo que va a constituir la maqueta y armazón simbólica del niño como sujeto inscrito en la historia familiar.

Debemos igualmente favorecer los intercambios madre-niño habituales y normales: palabras, caricias, sonrisas, contactos etc; así como favorecer salvo contraindicaciones precisas, el que el bebé pueda tener sus objetos transicionales e intermediarios (osito, chupete, etc).

Esta presencia e intercambios de los padres no debe reducirse a formas mecánicas (voz, tocar, mirar....) sino que debe favorecerse un modo de presencia multisensorial donde puedan "sentir su bebé", en una dimensión, no reducida a lo físico-corporal, sino en la construcción simbólica y de plenitud que se da en las condiciones normales de las relaciones afectivas primarias. En este sentido, debemos entender que la palabra tiene una función simbolizante y que el niño está preinscrito en el lenguaje que le preexiste a su nacimiento. Es en esta óptica, y desde esta función, como abordaremos toda interactuación y toda referencia a lo interactivo, sin reducionismos a los signos o señales basados en teorías de la acción-reacción .

Derivado de todo lo anterior, deben favorecerse estos reencuentros y la restitución de la maternidad y paternidad, siempre desde lo personalizado y especifico de cada caso: “... la importancia de las diferencias interindividuales hace pensar que una adaptación del entorno personalizado a cada niño será preferible a los protocolos universales ...." (V. Gramboulan). Una generalización abstracta supone ir en contra de lo particular y subjetivo que constituye la historia personal y familiar, derivado de las formas culturales y sociales, las costumbres, etc que componen los intercambios y la realidad humana.

\section{- A nivel institucional:}

El personal asistencial es un elemento clave, articulador de los cuidados técnicos y de los intercambios afectivos con los R.N. y su familia. Es preciso por tanto tener 
INFORMES

en cuenta algunos aspectos fundamentales de lo que está en juego en estos casos que van mas allá de los cuidados estricta y específicamente orgánicos, haciendo incluso que estos sean más eficaces.

Partiremos de que el niño es un ser bio-psico-social, que no se agota ni se reduce a uno de estos elementos, sino que para su armónico desarrollo requiere los lazos e intercambios afectivos y sociales que le permitan constituirse como ser humano.

La situación en que se encuentra el niño en estos casos, supone para los padres una quiebra de las ilusiones y aspiraciones puestas en su hijo que ahora se confronta a lo incierto de su enfermedad y a las angustias de muerte. Es por tanto fundamental que el equipo de cuidados puedan sostener, apoyar y elaborar estas vivencias.

Los padres van a confrontar al personal institucional a todo tipo de quejas, sumisiones, proyecciones, reinvidincaciones, rechazos e intercambios (desde sentirse padres indispensables a delegar en el personal toda función, desde sentirse sumisos y colaboradores a estar celosos, rivalizar o culpabilizar, etc). Es el personal quien va a sostener y recibir las ansiedades y angustias, las incertidumbre, las identificaciones y proyecciones que los padres van a depositar en ellos.

Es por tanto muy necesario poder constituir un medio lo más acogedor y favorecedor para el niño, los padres y el propio personal. De aquí la necesidad de realizar suspervisiones de casos y reuniones o apoyo psicológico que permitan manejar y elaborar estas problemáticas que serán fundamentales para una actuación global sobre el caso.

A este nivel institucional no querría dejar de señalar finalmente la necesidad de que existan comisiones de bioética que puedan intervenir desde una visión interdisciplinar y básica, en aquellos casos que por las distintas implicaciones del caso se precise de su opinión, máxime en un campo como el que abordamos, donde lo técnico, lo personal y lo social, están en permanente interelación y donde la dimensión ética adquiere especial relevancia.

\section{Resultados y conclusión}

Como puede apreciarse en las tablas expuestas, se realizaron una serie de intervenciones que nos permitieron cotejar nuestra observación de la expresión sintomática y las formas de reacción de los padres, que no difieren sustancialmente de lo que ya otros trabajos, especialmente psicodinámicos, nos han mostrado.

Dentro de lo que hemos denominado Programa Padres-Niño, se han realizado actuaciones tanto sobre el bebé como sobre la familia, los cuales hemos expuesto en la tabla 4, siendo la media de intervenciones entre 4 a 7 sesiones.

Se realizaron derivaciones a otros servicios de Salud Mental o bien de Atención Temprana, en aquellos casos donde la continuidad terapéutica requería un seguimiento y control mas específico. 
Asimismo en todos los casos se hizo un trabajo de observación del bebé y de supervisión y coordinación con el personal asistencial, para elaborar y manejar las distintas problemáticas que surgían en torno al niño y su familia, o bien como apoyo a los distintos problemas que aparecieron en la dinámica relacional y subjetiva.

Como conclusión podemos señalar, la importancia de una visión de trabajo en equipo e interdisciplinar al abordar este tipo de problemas en el bebé, y el papel de las intervenciones desde Salud Mental Infantil que permitan una actuación sobre el niño, la familia y a nivel institucional.

\section{TABLA 1}

UNIDAD SALUD MENTAL INFANTIL

$\mathrm{N}^{\mathrm{o}}$ de casos:

AÑO: $1.996=12$ casos

EDAD:

$$
\begin{aligned}
& 1 \text { a } 5 \text { días }=4 \\
& 5 \text { a } 15 \text { días }=3 \\
& 15 \text { a } 1 \text { mes }=1 \\
& 1 \text { a } 2 \text { mes }=1 \\
& 2 \text { a } 3 \text { mes }=2 \\
& \text { fallecido }=1
\end{aligned}
$$

FRATRIA:

Unico $=6$

2 hermanos $=2$

3 hermanos $=1$

$\mathrm{n} / \mathrm{s} \quad=3$

PROBLEMÁTICA SOCIAL $=3$

PADRES:

Biparental $=9$

Monoparental $=3$

( madre soltera)

ANTECEDENTES PSIQUIATRICOS:

$$
\begin{aligned}
& \text { Madre }=3 \\
& \text { Padre }=1
\end{aligned}
$$

TABLA 2
(Abandono - Maltrato...)
Cuadro clínico del bebé:

- Enfisema lobar congénito $\quad=1$

- Síndrome de Down $\quad=3$

- Cuadro derpivativo Social $=3$

- Atrofia Espinal

(Werdning Hoffmann) $=1$
- Síndrome de West $\quad=1$

- Sufrimiento fetal agudo $\quad=1$

- Síndrome Depresión Postparto

$$
\text { materto }=1
$$

- Mielomeningocele $\quad=1$ (fallecido) 
INFORMES

TABLA 3

CUADRO PSIQUICO REACTIVO EN LA MADRE:

Depresión $\quad=7$

Ansiedad $=5$

EXPRESIÓN SINTOMÁTICA Y VIVENCIAL :

. Sentimiento de culpa $=8$

. Rechazo $=8$

. Deseos de muerte $\quad=5$

. Venir fuera de lugar $\quad=3$

. Dificualtades a la relación $=11$

. "Es" enfermedad $=6$

. Prejuicio y desinformación $=5$

. Un castigo $=4$

. Incapacidad, Impotencia $=7$

. Ambivalencia Madre $\quad=10$

TABLA 4

ACTUACION PROGRAMA PADRES/NIÑO.

Psicoterapia

$=10$

Medicación

$=3$

Observaciòn Bebé

$=10$

TIEMPO DE ACTUACIÓN

$\begin{array}{ll}1 \text { Sesión } & =1 \\ 2 \text { a } 4 \text { Sesiones } & =4 \\ 5 \text { a } 7 \text { Sesiones } & =4 \\ 8 \text { ó más Sesiones } & =3\end{array}$

DERIVACIÓN

S.M. Adultos $\quad=5$

Atención Temprana $=6$

Otros Servicios = 3 


\section{BIBLIOGRAFIA}

- Ansermet F. Du bèbé néoténique au sujet. Notes critiques sur la psychiatrie du nourrisson Mental, 1. Juin 1995; 23-37

- Boris NW, Abraham J. "Psychiatric consultation to the neonatal intensive care unit: liason matters". J Am Acad Child Adolesc Psychiatry, 1999, oct; 38 (10); 1310-2

- Corominas R, Bayo C. "Relación entre R.N. de alto riesgo y sus padres". Rev. Asoc. Esp Neuropsiq, 1985; 14; 330-34

- Granboulan V, Danan C et al. "Prise en charge psychique de 1'extreme prematurité" Arch Pediatr. 1995; 2; 473-80

- Jellinek M.S etal. "Facing tragic decisions with parents in the neonatal intensive care unit: clinical perspectives". Pediatrics, 1992, Jan; 89 (1): 119-22

- Kreisler L, Bouchard F, et al . "Devenir psychologique des nouveau - nés traités en unité de soins intensifs". Ann Pediatr, 1978; 25; 509-18

- Lacan J. "Función y campo de la palabra y del lenguaje en psicoánalisis". Escritos. tomo I. Madrid. Siglo Veintiuno editores. 1978.

- Mira, V. "Suposición del sujeto en el psicoanálisis de niños". Rev. Asoc Esp Neuropsiq $1985 ; 14 ; 322-29$

- Morandé G. y Bayo C, Anta C. “Grupos Parque”. Rev Asoc Esp Neuropsiq 1984; 11; 357-62

- Pallas Alonso CR et al. "El ambiente en los cuidados intensivos neonatales". An. Esp. Pediat. 1997.; 47 ;618-20

- Pedreira J.L “Aspectos psicosociales de los ingresos en neonatología”. XXX II Reunión de la Soc Esp de Psiquiatría Infanto-Juvenil. Valencia. Mayo 1993.

- Soulé M. "Les souhaits de mort en pediatrie du nouveau-nés, comprehensión de la dynamique confluctuelle chez les parents de l'enfant prématuré et dans l'equipe soignante".

Rev Neuropsychiatr Infant Hyg Ment Enfance; 1978; 26; 439-49

- Winnicott W. La theorie de la relation parent-nourrison et l 'observatión des jeunes enfants dans une situation etablie. De la Pediatrie a la Psychanalyse. Paris. Payot 1971.

\footnotetext{
* Psiquiatra Infantil - Unidad de Salud Mental Infantil

Complejo Hospitalario Juan Canalejo - A Coruña
}

Fecha de recepción: 4-V-2000 